\title{
Visllani Visllacuni: Patrones de Consumo a Comienzos del Horizonte Medio
}

\author{
"What was served with these spoons? Patterns of \\ Consumption at the beginning of the Peruvian \\ Middle Horizon
}

ANITA G. CooK

\section{RESUMEN}

"Visllani visllacuni", frase quechua que titula nuestro trabajo, significa: ¿Qué servían con estas cucharas?Este estudio trata de la producción local de cucharas pintadas y modeladas de distintas formas y tamaños, procedentes de las excavaciones en Conchopata, Ayacucho, con el fin de entender cómo las cucharas fueron utilizadas en la práctica cotidiana. El reconocimiento de patrones de producción, circulación y consumo sugieren que las cucharas pueden revelar comportamientos domésticos y rituales que no han sido abordados en la literatura.

Generalmente las cucharas no son consideradas objetos especiales o como objetos con dimensiones simbólicas, sin embargo, durante el Horizonte Medio (AD 550-1000), en el valle de Ayacucho en los Andes centrales, se han identificado cucharas que fueron recuperadas no sólo desde áreas domésticas, sino más bien de una variedad de contextos. Entre éstas, se han identificado tres tamaños de cucharas. Aqui nos enfocamos en la procedencia, diseño y el modelado de las cucharas en la categoría de menor tamaño. Se considera que las cucharas tenian no solo unas funciones utilitarias sino que conte-

1 The Catholic University of America. E-mail: cook@cua.edu

Recibido: Enero 2008. Aceptado: Abril 2008. 
nian un significado simbólico para actividades funerarias y otros usos rituales festivos.

Palabras Claves: Imperio Huari/Wari, cucharas, producción de cerámica, contextos rituales y domésticos.

\section{Abstract}

"Visllani visllacuni" the Quechua expression in the title means "what did they serve with these spoons?" This study examines locally made spoons in distinct shapes and sizes from excavations at the site of Conchopata, Ayacucho, in order to understand how spoons were used in daily practice. Notable patterns of production, circulation and consumption strongly suggest that spoons can both reveal domestic and ritual behaviors which have not previously been addressed in the literature.

Spoons are not generally examined as luxury goods or as objects with symbolic dimensions, yet during the early Huari Empire ( $A D$ 550-1000) in the central Andean Valley of Ayacucho spoons were excavated not only from expected domestic areas, but from a variety of contexts. Three spoon sizes are identified. In this essay, I focus on the provenience, design and modeling of the smallest size category. Although spoons had a practical use they also had symbolic significance that transcended life and is expressed in their association with the dead, and associated rituals.

Key words: Huari/Wari Empire, spoons, ceramic production, domestic/ritual consumption

\section{INTRODUCCIÓN}

"...entre los objetos del rescate de Atahuallpa, que Francisco Pizarro envió de regalo al rey Carlos $V$, se nombran vasijas, platos, ollas, botellas, fuentes, cucharas y cántaros de oro y plata" (Relación Francesa de la Conquista del Perú 1968 [1543] t. I: 184-187, citado por Olivas 2001: 69).

"[Las cucharas incas]... no tenian la forma universalmente conocida, sino que eran casi rectangulares, convexas por fuera y cóncavas por dentro, de arcilla color ocre rojizo y carecian de decoraciones. Las habia en tres tamaños, que correspondian a las edades de los 
comensales; es decir, viejos, jóvenes y niños» (González Holguin 1989 [1608]: 140, 354, 466, citado por Olivas 2001: 72).

¿Qué importancia tiene un estudio de cucharas pre-incaicas como tema de este artículo? Llegué al estudio de estos objetos, que reconocemos como cucharas, a través de nuestras investigaciones sobre la organización de producción cerámica en Conchopata, un sitio arqueológico que ha sido identificado anteriormente por varios arqueólogos (Benavides 1965; Lumbreras 1974; Pozzi-Escot 1991; Pérez 1998; Pérez y Ochatoma 1998; Ochatoma y Cabrera 2000; etc.) como un centro de producción alfarero.

La principal interpretación de estos investigadores y gran parte de la literatura sobre este sitio, han enfatizado el rol económico: es decir, que Conchopata era un pueblo de alfareros. Estos mismos colegas también reconocen que los alfareros produjeron un amplio rango de vasijas de gran tamaño hasta en miniaturas y posiblemente de muchos estilos típicos del Horizonte Medio. El objeto del Proyecto Arqueológico Conchopata (PAC), que aún se desarrolla, es entender el rol que el sitio tenía a través de su historia. ¿¿Fue solamente un pueblo de artesanos? ¿Quiénes y por cuánto tiempo habitaron este sitio?

La ciudad de Huari se ubica a unos doce kilómetros al norte del valle de Ayacucho y podemos afirmar que durante el Horizonte Medio (5501000 d.C.), Conchopata fue la comunidad más importante al sur del mismo valle (Figura 1).

En esta ocasión presento específicamente un artículo sobre cucharas de cerámica encontradas mayormente en el valle de Ayacucho durante el Horizonte Medio, con la finalidad de entender el contexto, uso, circulación y deposición de este tipo de artefacto, en otras palabras, la historia cultural (Gosden y Marshall 1999) y la chaine operatoire (cadena operativa) de estos objetos. Las cucharas representan artefactos que fueron producidos con mucho cuidado, finamente pintadas y muchas veces con los extremos de los mangos decorados con efigies o esculturas de animales o seres antropomorfos en miniatura (Figura 2). 


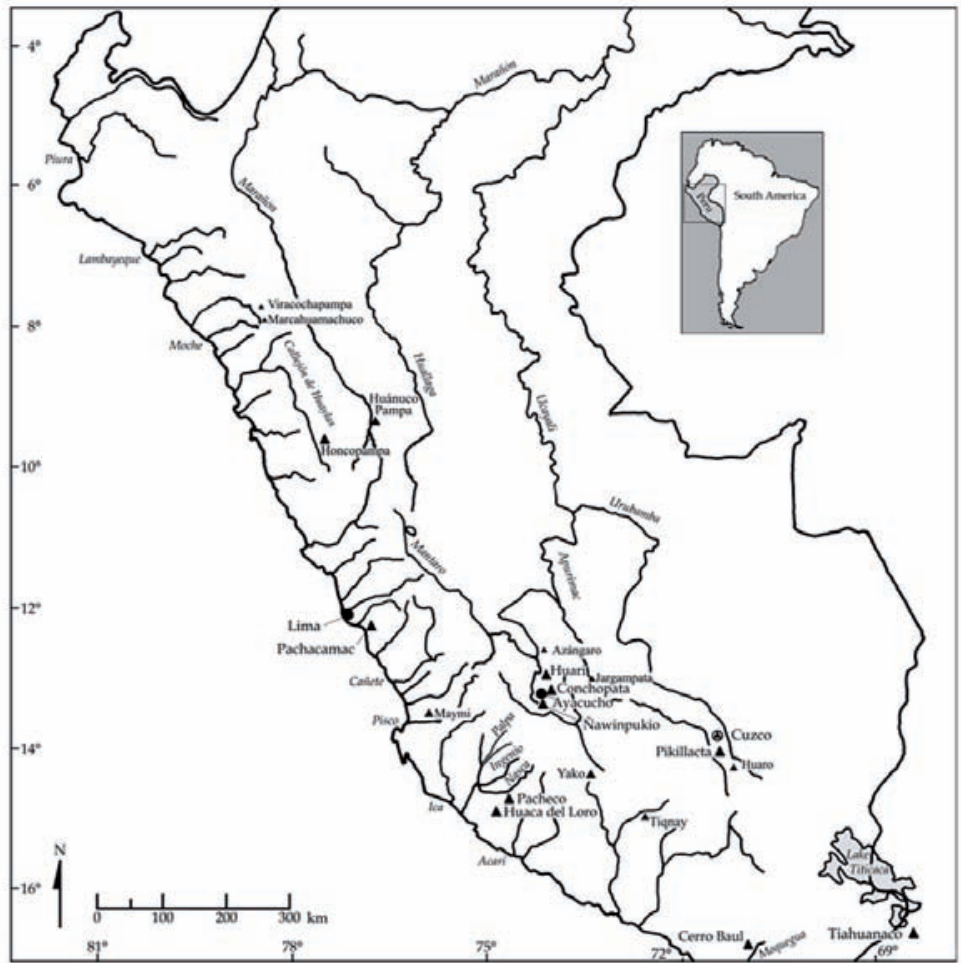

Figura 1. Mapa de Ayacucho con la ubicación del sitio de Conchopata.

Figure 1. Map of Ayacucho including the location of the site of Conchopata.

Existen distintas interpretaciones recientes sobre el sitio de Conchopata, por ejemplo, Ochatoma y Cabrera enfatizan la cerámica ritual de Conchopata, particularmente aquellas encontradas en una estructura en forma de D, pero consideran el sitio como un pueblo de artesanos. Isbell (2001) propone que es un palacio en una zona urbana donde se produjo cerámica para la elite, y que la gran cantidad de urnas y cara-golletes con iconografía, representan la vajilla de una nueva generación de dirigentes. Al fallecer éstos, se rompía ritualmente la cerámica del reino anterior. Es una interpretación interesante pero las evidencias no permiten comprobarla. ¿Cómo podemos averiguar si las urnas rotas intencionalmente no se hicieron cuando el sitio fue destruido por los mismos pueblos de Huamanga, a la caída del imperio Wari?

La autora del presente trabajo reconoce que el sitio era uno de los pueblos más importantes del valle desde fines del Periodo Intermedio Temprano hasta fines del Horizonte Medio. Esta importancia es evidente por la presencia de arquitectura doméstica para familias extendidas, edificios para los gobernadores o elites intermediarias, templos con patios y estructuras 
rectangulares, con patios internos identificados como patio y galería, arquitectura funeraria y avenidas. No se encuentran lugares obvios para almacenamiento pero sí cuartos llenos de vasijas grandes para almacenar agua y comida al nivel doméstico. La perspectiva de que una gran parte de la excavación del Proyecto Arqueológico Conchopata (PAC) fue un palacio, como lo define Isbell (2001), se basa en rasgos compartidos por algunos sitios wari, y no define específicamente una residencia de las elites. Similarmente, no aborda el problema de cómo fueron gobernados los pueblos bajo la hegemonía wari.
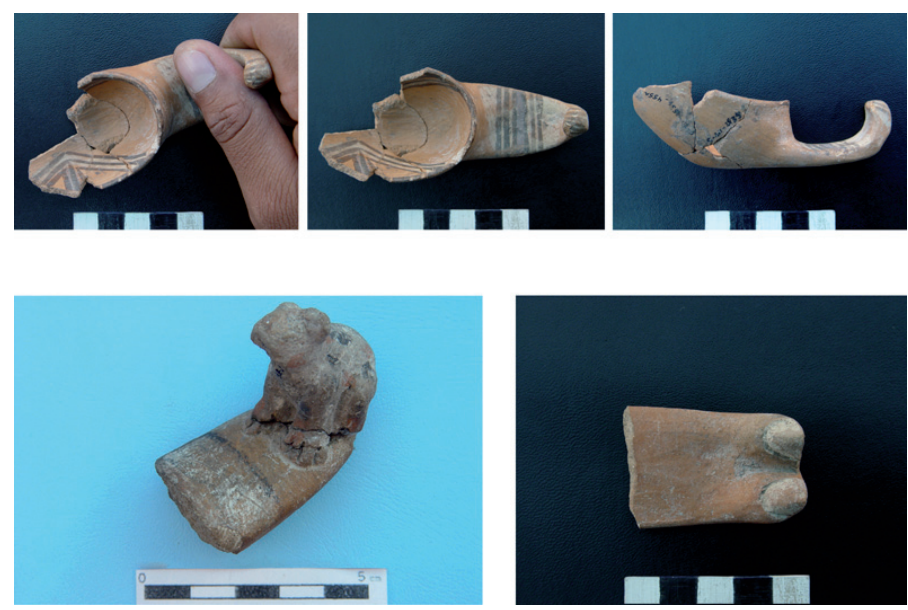

Figura 2. La diversidad de cucharas decoradas de Conchopata. Primer fila: tres vistas de una cuchara del Espacio Arquitectónico 211. Segunda fila: cuchara con felino, Espacio Arquitectónico 106; Mango en forma de pata de llama, Espacio Arquitectónico 175 T1.

Figure 2. Variations of decorated Conchopata spoons. Upper row three views of a spoon from Room 211. Lower row handle with feline from Room 106, handle in the form of a llama foot from Room $175 T 1$.

Las estructuras más conocidas como patio y galerías (Figura 3), donde los restos excavados en su interior y adyacentes sugieren que hubo fiestas o festines en su patios, y el almacenamiento y preparación de comidas y bebidas en las galerías (Brewster-Wray 1983, 1990). En el patio de estos edificios se encuentra una cantidad mayor de cuencos pequeños, keros y vasos, además de la presencia de urnas y enormes jarras para la comida y bebida servidas (Cook y Glowacki 2003). Estos datos sustentan aún más la interpretación de Brewster-Wray (1990), que estos recintos rectangulares eran los patios centrales de las residencias de elite en la ciudad de Huari, un modelo que es relevante a otros sitios wari que comparten el mismo tipo de arquitectura. 
Los entierros en Conchopata tienen una historia compleja que incluye una variedad de tumbas y áreas dedicatorias que subrayan la importancia del sitio a través del tiempo (Tung y Cook 2006), y el deseo de ser enterrado en el hogar. También se encuentran entierros secundarios que indican que éstos fueron removidos y quizá traídos al sitio (Isbell y Cook 2002; Isbell 2004).

En un estudio de enterramientos de restos humanos, Tung (2003) y Tung y Cook (2006) han sugerido que existe una desviación demográfica en Conchopata y que los entierros reflejan distintas clases sociales. Hay casi dos veces más mujeres que hombres enterrados en el sitio. Hay también muchos jóvenes así como recién nacidos. Hasta ahora no hemos podido explicar el lugar donde enterraron a los hombres de Conchopata o cómo trataron el cuerpo masculino después de la muerte. Lo que es evidente es que aquí vivían muchas mujeres con sus hijos. También hay evidencia de sacrificios humanos en forma cabezas trofeos (Tung 2003), en arquitectura cívica o de carácter no domestico como las estructuras en forma de D. Estos datos nos llevan a considerar que Conchopata no pudo haber sido solamente un sitio de producción intensiva de cerámica, sino también un sitio de elites intermediarias, muchas de ellas de mujeres, y que varias actividades habrían ocurrido en el sitio.

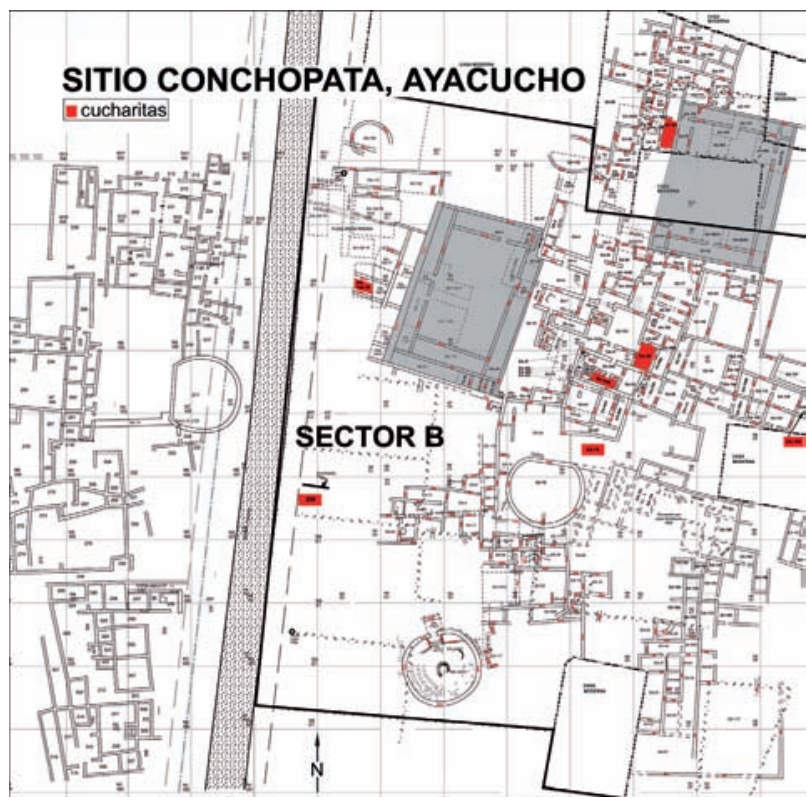

Figura 3. Plano del sitio de Conchopata. Patio y galerías en gris, y ubicación de las cucharitas en rojo.

Figure 3. Map of the site of Conchopata indicating patio and galleries in grey, and the distribution of small spoons in red. 
Propongo que sitios wari que incluyen el típico patrón de arquitectura compleja de patio y galerías (Figura 3) (frecuentemente acompañado por estructuras de adoratorios en forma de D y espacios domésticos), representan los vestigios de edificios que comprendían actividades múltiples, y que los habitantes pudieron haber usado los patios y galerías de manera distinta, de una estación a otra. Para observar cómo se identifican estas diferentes actividades estamos analizando la distribución de artefactos excavados.

Un ejemplo que nos ayudó a identificar zonas de posible producción artesanal fue las altas concentraciones de distintos tipos de herramientas de producción alfarera. El problema que surgió es que estos mismos espacios no son zonas anteriormente consideradas en la literatura como espacios de actividad artesanal. En las excavaciones que realizamos en Conchopata hemos encontrado abundante material que contradice muchas presunciones. Por ejemplo, el sitio demuestra una complejidad arquitectónica que sugiere a primera vista una división entre espacios cívicos-religiosos y espacios domésticos. Sin embargo, cuando Cook y Benco (2001) analizaron la distribución de artefactos de producción alfarera, los resultados indicaron que ambos tipos de espacio también habían sido utilizados para distintas etapas de producción alfarera. Esto sugiere que la producción alfarera ocurrió no solo al nivel doméstico, sino también por especialistas que podrían haber utilizado ciertos edificios para talleres.

Cuando nos preguntamos si se desarrollaron festines en los patios y galerías, organizados por el estado o imperio, también encontramos que existen datos suficientes para esta interpretación. Lo curioso es que los espacios artesanales se encuentran también en los mismos espacios identificados como zonas donde hubo evidencia de comidas al nivel comunitario o festines. Evidencias de comidas especiales en las que muchas personas participaron, coinciden con zonas ideales para la preparación y construcción de vasijas ceremoniales. Entonces, ¿cómo se explica esta coincidencia? ¿Estamos seleccionando los datos que se necesitan para verificar una interpretación premeditada? Quizás, pero es más probable que espacios amplios como patios y plazas, fueran utilizados para diferentes actividades. Sin embargo no se puede pasar por alto el que los espacios arquitectónicos excavados por el PAC tienen mucho relleno y que hubo bastante predeposición de materiales en el sitio desde tiempos antiguos. Las pocas zonas de deposición primaria existente, es lo que queda de la destrucción actual del sitio que hoy continúa, además de la destrucción y huaqueos antiguos. 


\section{Las CUCHaras de Conchopata}

En este artículo quiero llamar la atención a otros indicadores de uso espacial, es decir, la frecuente presencia de «cucharas» de cerámica en espacios de uso múltiple. Se supone que las cucharas, al igual que otros implementos que se utilizaban para servir o consumir alimentos y otros usos cotidianos de carácter doméstico, fueron hechas de materiales orgánicos como madera y hueso, los que no se conservan bien en los depósitos arqueológicos de la sierra. Por lo tanto, la frecuencia y distribución de cucharas de cerámica tuvieron un rol quizás distinto o de prestigio en comparación a otros materiales más desechables. Lo que es importante observar es que no todas las sociedades de los Andes han tenido la costumbre de producir y/o usar cucharas de cerámica.

Las cucharas fueron bastante conocidas en la sierra y costa norte durante el Período Intermedio Temprano, en sitios de filiación cajamarca y recuay; también Moche tiene su variedad de cucharones, pero cucharas de cerámica no son tan frecuentemente observadas en otras sociedades prehispánicas. Se observan generalmente asociadas a conjuntos de parafernalia alucinógena en Moquegua y Tiwanaku, y en el norte de Chile con la iconografía de la Portada del Sol en Tiwanaku. Es interesante notar que gran parte de las cucharas en el sur son hechas de madera.

Las cucharas de Conchopata, como se verá más adelante, no se identifican con la iconografía del Altiplano. Si las relaciones entre la sierra central de Ayacucho y el Altiplano fueron tan fuertes como indica la iconografía que incluye el Dios con los báculos y figuras asociadas, el uso de cucharas debe haber tenido otro origen. Además, la costumbre de producir cucharas de cerámica es intencional y con fines muy específicos. No tenemos aún datos sobre el proceso u organización de la producción de cucharas en Conchopata, pero este estudio me ha convencido que es necesario apuntar en esa dirección.

\section{LA MUESTRA Y LOS ESTILOS}

La primera parte de este estudio fue realizada en el año 2004 mediante el análisis de hasta entonces 74 cucharas, y a la fecha hemos analizado 641, y es lo que aquí presentamos. Los resultados preliminares indicaron una mayoría de cucharas del estilo Huarpa del Periodo Intermedio Temprano. 
CUCHARAS: Distribución por estilo y tamaño

$(\mathrm{N}=641)$

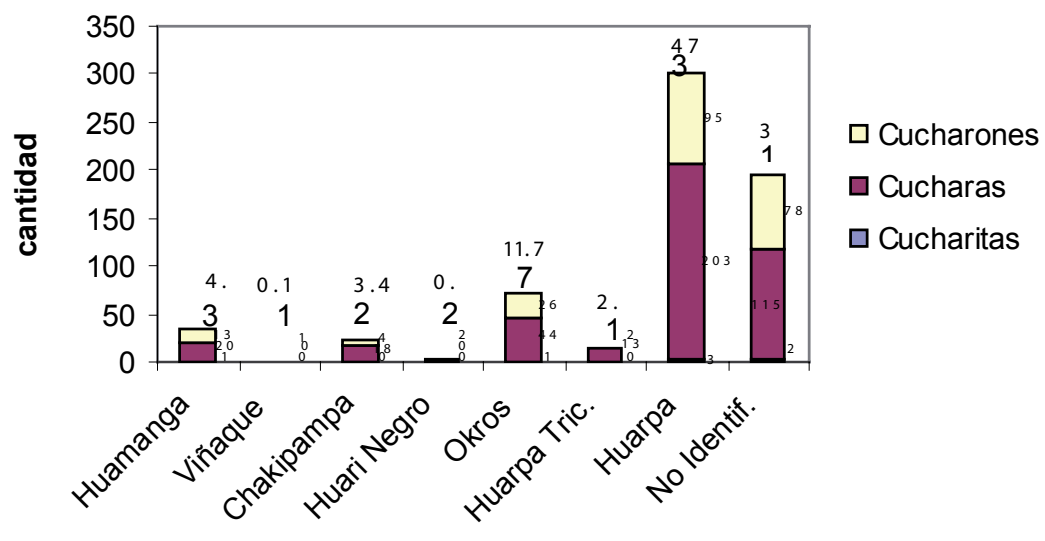

Figura 4. Histograma de cucharas: distribución por estilo y tamaño.

Figure 4. Histogram of spoons by style and size.

De las 641 cucharas analizadas (Figura 4), la mitad de la muestra es del estilo Huarpa (49\%). Los resultados de las excavaciones (Leoni 2004) en el sitio Nawinpukio, a unos 5 kilómetros al sur de Conchopata (Leoni 2004), indican que el estilo Huarpa continúa durante el Horizonte Medio temprano. Entre las cucharas encontradas en Conchopata, 11,7\% corresponden al estilo Okros, y 4,8\% corresponden al estilo Huamanga. Le sigue el estilo Chakipampa con el 3,4 \% ${ }^{2}$. Por lo tanto, es acertado reconocer que Huarpa se mantiene como el estilo principal. Esto sugiere que antes del desarrollo de los estilos asociados con Wari, el uso de la cuchara era frecuente $\mathrm{y}$ formaba parte de las costumbres locales.

¿Con qué propósitos se utilizaron estas cucharas, muchas de ellas finamente decoradas?

\section{TAMAÑO Y TIPOS DE CUCHARAS}

La mayoría de las cucharas están fracturadas o rotas por lo menos dos veces, y solo hay muy pocas completas. El análisis inicial basado en una muestra de 74 cucharas indicó que la mayoría son cucharitas que miden entre 3 a 6 centímetros de largo. En el análisis preliminar tomé en cuenta solo el largo del fragmento, pero en realidad esta observación refleja el hecho de que la muestra está mayormente compuesta de fragmentos. Luego se determinó que el ancho del mango (Figura 5) de una cuchara resultaba ser mucho 
más indicativo del tipo de cuchara cuando se trata de artefactos incompletos (Huamán 2005). Esto alteró la interpretación anterior. Por lo tanto, esta nueva forma de clasificar el tamaño dio lugar a una distribución que es muy diferente (Figura 6): 7 cucharitas (6,19 centímetros de largo), 417 cucharas (6,5-10 centímetros) y 217 cucharones (10,5-20 centímetros).

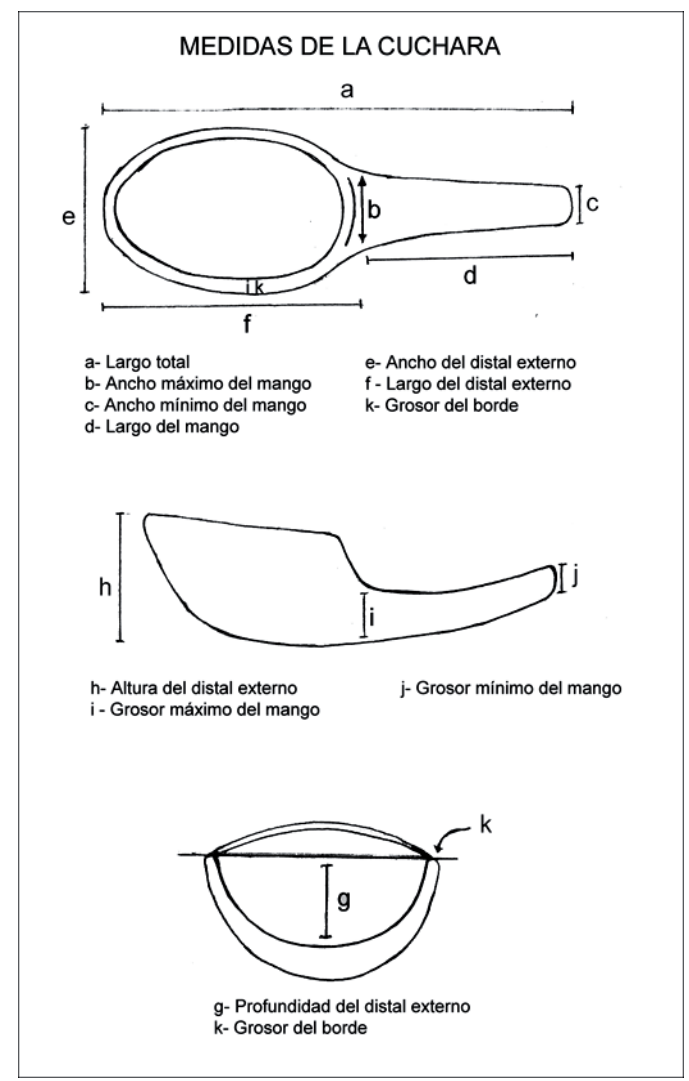

Figura 5. Medidas de la cuchara.

Figure 5. Spoon measurements.

Hay varias formas de cucharas, cucharitas y cucharones, y en muchos casos, el mango es el punto más débil o donde se encuentra la fractura del fragmento. Tomando medidas precisas de las cucharas completas, de las casi completas y del ancho de los mangos, resulta que hay un promedio de tres tamaños de cucharas: pequeñas, medianas y grandes. Como mencionamos antes, las denominamos cucharitas, cucharas y cucharones. Esta distinción no significa que las funciones modernas de estos objetos se aplican directamente a las sociedades Huarpa y Wari, como veremos en lo que sigue.

La distribución espacial de cucharitas (Figura 7), sin contar la superficie y las dos primeras capas más disturbadas, A y B, demuestran que 
son muy escasas y aparecen principalmente en tumbas y cistas de cerámica a manera de ofrenda (Figura 3). Mientras que las cucharas y cucharones representan la mayoría de los fragmentos, hay dos veces más la cantidad de cucharas, lo cual podría representar un uso más cotidiano, con cucharas para uso particular (Figuras 8-10) y cucharones (Figura 11) para servir.

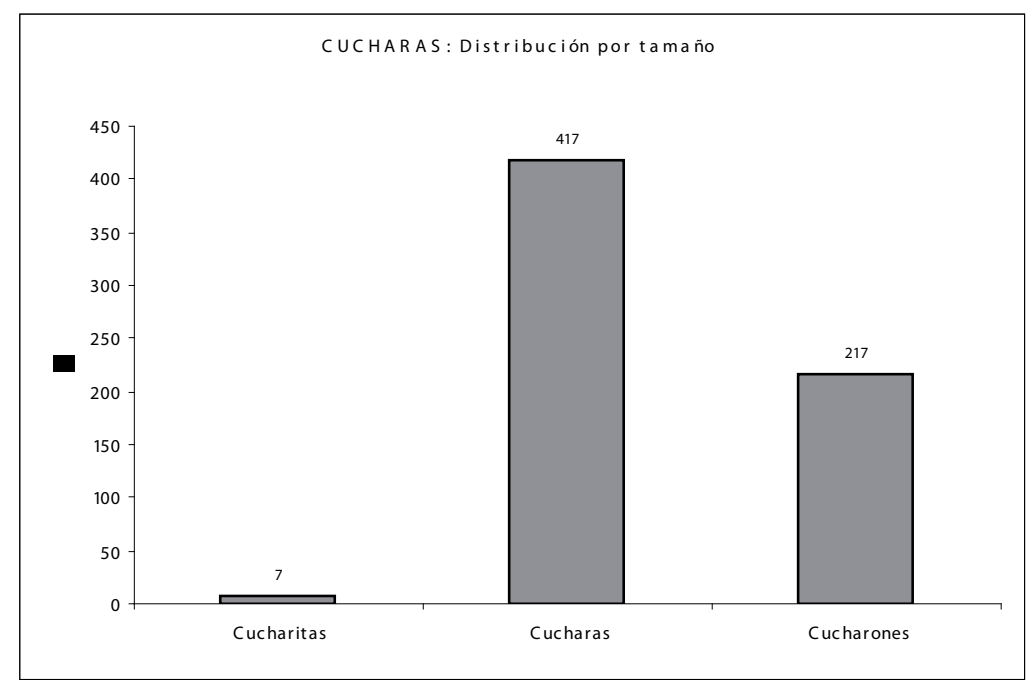

Figura 6. Cucharas: distribución por tamaño.

Figure 6. Distribution of spoons by size (small, medium, and large).

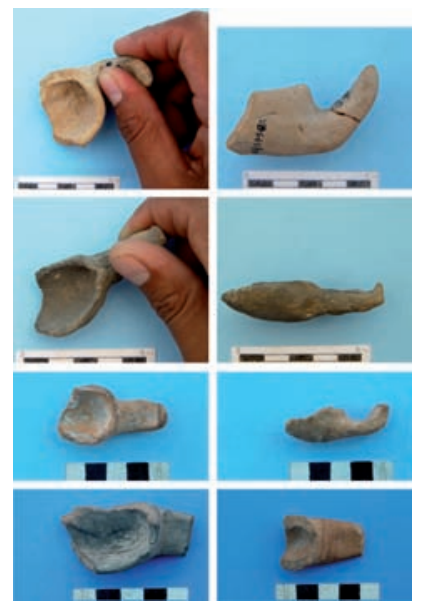

Figura 7. Cucharitas. Primera fila: Espacio Arquitectónico 104 T6. Segunda fila: Espacio Arquitectónico 208. Tercera fila: Espacio Arquitectónico 75.

Cuarta fila: Espacio Arquitectónico $44 B$ y 90.

Figure 7. Small spoons. Top row from Room 104T 6. Second row from Room 208; Third Row from Room 75; Bottom Row from Room 44B and 90. 
Para contribuir a su estudio también recolectamos información sobre las variables de desgaste a fin de averiguar cómo se usaron las cucharas y su relación al uso de vasijas para servir. Aquí quiero enfatizar que la constante presencia de huellas de uso o desgaste y de re-uso sugiere que las cucharas fueron usadas frecuentemente, o alternativamente por mucho tiempo, pero sólo en ocasiones especiales. Existen muestras de cucharas fragmentadas que llevan perforaciones, las que probablemente fueron hechas para "coserlas» a manera de repararlas. En las conclusiones sugiero cuál de las dos posibilidades nos parece más factible. Todo esto nos conducirá a una interpretación más amplia sobre el uso del espacio en Conchopata y las actividades rituales, domésticas, festivas y en los momentos funerarios.

\section{INTERPRETACIÓN DE LAS CUCHARAS: LA EXPERIENCIA VIVIDA}

El estudio de cucharas de madera que llevan la iconografía de la Portada del Sol en Tiwanaku (p.e. el Dios con los báculos y figuras asociadas), es decir, temas iconográficos del Altiplano en combinación con diseños de carácter local, han sido encontrados en contextos funerarios del área sureña en San Pedro de Atacama, Chile. Las condiciones áridas conservaron una variedad de cucharas a través del tiempo. Espoueys (1972-1973: 70) sugiere algunas funciones principales para este utensilio: 1) como uso ritual, 2) para la transferencia de líquidos, polvos o granos, y 3) función comestible que puede incluir el mezclar y servir comida / bebidas. Espoueys sugiere que el tamaño y forma de la cuchara dicta su función más apropiada. Enfocándonos solamente en las siete cucharitas y en las cucharas con un mango finamente modelado (Figuras 2, 10) de Conchopata, ¿es posible establecer la función de estas cucharas a través de su forma y tamaño como lo sugiere Espoueys? Consideramos también las referencias de la época colonial mencionadas al inicio de este trabajo: «...que había [cucharas] en tres tamaños, que correspondían a las edades de los comensales; es decir, viejos, jóvenes y niños...» (Pardo 1957: 590-591; González Holguín 1989 [1608]: 140, 354, 466, citado por Olivas 2001: 72). 


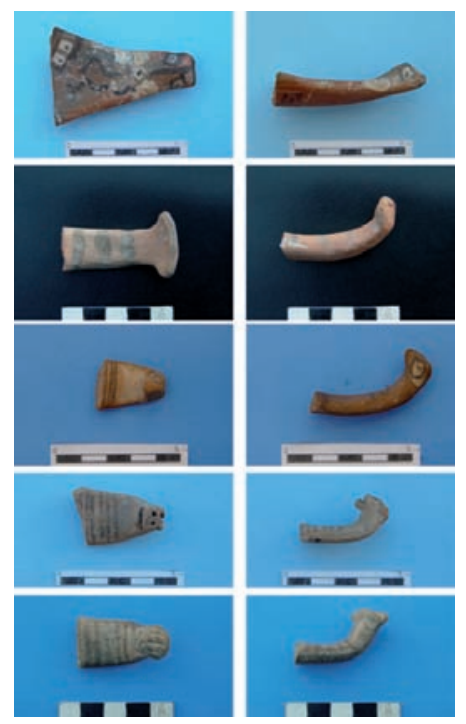

Figura 8. Cucharas. Espacios arquitectónicos 41, 146, 6, 20, 33.

Figure 8. Medium sized spoons: Room 41, 146, 6, 20, 33.

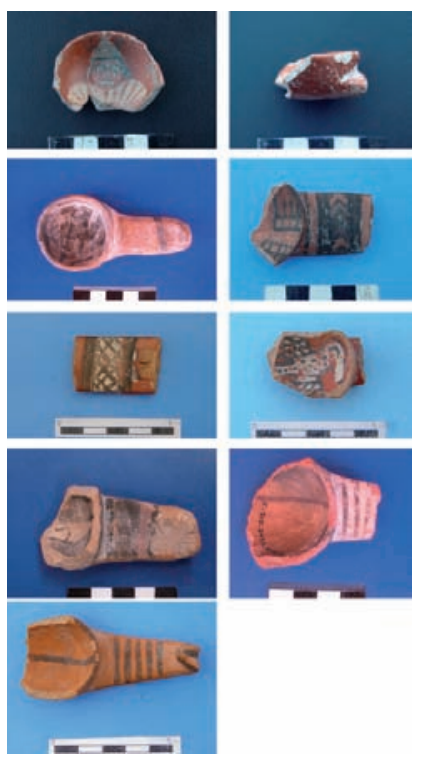

Figura 9. Cucharas. Primera fila: retrato de una figura humana, Espacio Arquitectónico 20. Segunda fila: Espacio Arquitectónico 40B, 33. Tercera fila: Espacio Arquitectónico 6, Espacio Arquitectónico 104 con figura de ave. Cuarta fila: Espacio Arquitectónico 14372 y 210, Espacio Arquitectónico

$$
1121 T 2 .
$$

Figure 9. Medium sized spoons. Top row human figure from Room 20. Second row from Room 40B, 33. Third row from Room 6, and 104 with bird design. Fourth row from Room $143 T 2$ and 210, and Fifth row from Room 1121 T1. 
Consideramos solo las cucharitas, y nos preguntamos: ¿por qué hay muy pocos ejemplos? Primero: ¿podemos intentar identificar los espacios arquitectónicos y sus contextos individuales para averiguar si encontramos un patrón de distribución que conlleve una posible interpretación? Los espacios arquitectónicos donde se encuentran las cucharitas están indicados en el plano de Conchopata (Figura 3), y se distribuyen en zonas donde hay concentración de entierros y cistas de cerámica de probable origen ritual o ceremonial.

Segundo: ¿sería posible que las cucharitas puedan haber sido utilizadas en tiempos tempranos, es decir en la sociedad Huarpa, quedando así pocos restos arqueológicos en nuestra muestra? Las cucharitas huarpa tienen mejores contextos que aquellas de otros estilos. Éstas provienen de un piso, un hoyo con cerámica y del interior de una banqueta. Otros estilos de cucharitas son Okros y Huamanga, con dos fragmentos que no fueron definidos estilísticamente. Si hubo una fase temprana del estilo Huamanga, como fue sugerido por varios arqueólogos (Anders 1986; Pozzi Escot 1991; Ochatoma y Cabrera 2000; Leoni 2004), entonces las cucharitas podrían ser parte de una tradición que empieza durante el Período Intermedio Temprano con la sociedad Huarpa, y continúa durante el Horizonte Medio temprano.
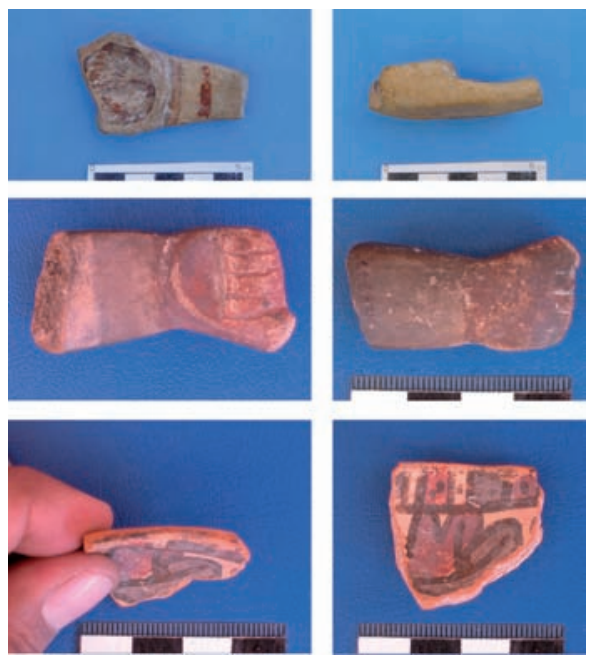

Figura 10. Cucharas. Primera fila: Espacio Arquitectónico 112T2. Segunda fila: Espacio Arquitectónico 181, puño en alto humano. Tercera fila: Espacio Arquitectónico 133.

Figure 10. Medium sized spoons. Top row from Room 112T2; Second row from Room 181 in the form of a human fist. Bottom row: Room 133. 


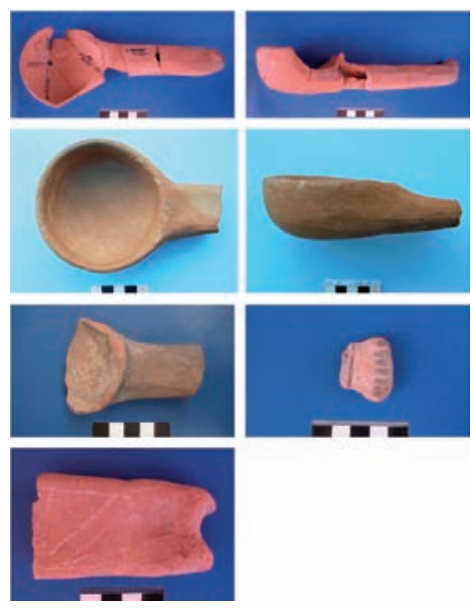

Figura 11. Cucharones. Primera fila: Espacio Arquitectónico 150. Segunda fila: Espacio Arquitectónico 31. Tercera fila: Espacio Arquitectónico 2, 80a. Cuarta fila: Espacio Arquitectónico 152T2.

Figure 11. Large spoons/ladles. Top row from Room 150. Second row from Room 31. Third row from Room 2 and 80a. Fourth row from Room 152T2.

Tercero. Dado el alto número de entierros de jóvenes en el sitio, ¿podemos considerar las cucharitas como juguetes o cubiertos de infantes? Siendo hechas de cerámica, estas se rompieron más fácilmente. La baja frecuencia de cucharitas sugiere que no fueron utilizadas como utensilios cotidianos para niños sino como objetos de prestigio y de uso en ocasiones funerarias y como ofrendas. Los contextos no proveen información en este sentido. Es probable que las cucharitas de madera fueran más prácticas para los niños.

Cuarto. Si las cucharitas son escasas y muy pequeñas, ¿podríamos considerarlas como miniaturas? El rol de las miniaturas en los Andes hasta nuestros días conlleva un sentido ritual y ceremonial. Tenemos dos contextos interesantes. El primero es en un espacio arquitectónico (EA 94) donde se encontró una cucharita huarpa dentro de un hoyo con más de 400 urnas en miniatura del estilo Horizonte Medio que fueron rotas intencionalmente. La cucharita huarpa pudo haber sido un precioso objeto ritual de herencia familiar. Por otro lado, cucharitas que nosotros consideramos en el estilo Huarpa podrían haber sido emuladas en tiempos más tardíos por las siguientes generaciones, quienes reprodujeron ejemplos en ocasiones rituales que rememoraban los ancestros abuelos, bisabuelos, etc.

El segundo contexto es el Espacio Arquitectónico 44b, que forma parte de una zona funeraria que incluye varios tipos de tumbas y cistas con 
restos humanos. Actualmente estamos en plenos estudios para determinar a los individuos enterrados en la tumbas de este espacio.

Por lo tanto, estas dos cucharitas ocurren en los contextos funerarios o cerca de ellos y en "ofrendas de miniaturas».

\section{Conclusiones}

El estudio de cucharas de madera prehispánicas tiene una larga historia. Por ejemplo, en el área sureña en San Pedro de Atacama, Chile, Espoueys (1972-1973: 70) sugiere algunas funciones principales para este utensilio: 1) como uso ritual, 2) para la transferencia de líquidos, polvos o granos, 3) función comestible que puede incluir mezclar y servir comida / bebidas. Él sugiere que el tamaño y forma de la cuchara dicta su función más apropiada. En la sierra central sabemos que sitios como Conchopata tenían una alta producción de objetos de cerámica y que era una actividad cotidiana durante ciertas épocas del año. Producir cucharas era una parte de la vajilla ayacuchana, especialmente a fines del Periodo Intermedio Temprano e inicios del Horizonte Medio. El hecho es que tenemos dos cucharas por cada cucharón (217 cucharones y 417 cucharas), lo que sugiere un uso práctico. Se supone que los cucharones eran más apropiados para servir mientras que las cucharas fueron usadas para consumir alimentos (sopas, estofados, papas, etc.) pero en festividades $\mathrm{u}$ ocasiones especiales. Las cucharitas y cucharas con mangos escultóricos o con efigies son muy escasas. Las cucharitas con efigies en buenos contextos son aún menos frecuentes, pero 3 se encuentran en el Espacio Arquitectónico 6 (EA-6), con un piso de arcilla roja. En la esquina noreste hubo una tumba fina pero disturbada. Otros artefactos y restos sugieren que pudo haber sido el patio de una residencia de alto status social (Isbell et al. 2002). Las cucharitas eran intencionalmente enterradas, es decir, en contextos no utilitarios.

En nuestra zona de excavaciones en Conchopata, tenemos tantas estructuras especiales que, como he sugerido en las primeras páginas, hay investigadores que prefieren interpretar este lugar como un sitio de especialistas en la producción de cerámica. Otros interpretan el sitio como un palacio (Isbell 2004) con zonas de residencias, salas de recepción, etc. Isbell ofrece, de una manera procesual, una lista de variables que pueden definir un palacio «andino». Puede ser que Pachacámac y Huari ofrezcan mejores indicadores para el Horizonte Medio, pero lamentablemente es difícil visualizar un palacio en Conchopata. Esto se debe en gran parte al hecho que mirando solo la cáscara, es decir, la arquitectura formal y el gran tamaño de 
unas tumbas vacías como enfatiza Isbell, pone de lado todo el contenido de cada espacio arquitectónico. No obstante el hecho que hay que tener en cuenta es que hubo deposiciones secundarias, y hay vestigios de actividades primarias en el sitio.

Hemos visto que las cucharitas de Conchopata cumplen varias funciones. Primero, estas parecen haber tenido un rol más ritual, siendo miniaturas, y encontradas en un deposito de urnas para miniaturas rotas intencionalmente. La baja frecuencia de éstas no sugiere que fueran utilizadas como juguetes y utensilios. Se supone que teniendo una amplia población de infantes en el sitio, el número de las cucharitas habría sido mayor de las que hemos encontrado. Segundo, la mayoría de las cucharitas, es decir, casi el 50\%, son del estilo Huarpa o Huarpa Tricolor, asociados con otros estilos a comienzos del Horizonte Medio. Lo interesante es que el uso de la cuchara por las poblaciones del valle de Ayacucho empezó durante el Periodo Intermedio Temprano, cuando ya existía contacto con la sierra norte.

La presencia y contexto de cucharas del Periodo Intermedio Temprano en la sierra norte ofrecen información paralela a lo que estamos observando en Conchopata. Joan Gero (2001: 22) nos indica que «en el Sector III del sitio de Queyash Alto, se ubicaron tres cucharones y cucharas con un abundante número de cántaros y vasijas pequeñas que fueron ideales para servir y tomar bebidas». T. Topic y J. Topic (1984) encontraron en sus excavaciones cucharas del estilo Cajamarca Cursivo y un cuenco con pedestal, como ofrendas en una tumba en el mausoleo del Horizonte Medio del Cerro Amaru, Huamachuco. Más recientemente J. Topic y T. Topic (2001) mencionan que:

"Desde hace años se sabe que la cerámica de Cajamarca estuvo muy difundida durante el Horizonte Medio. Además, las formas cerámicas cajamarquinas más difundidas son precisamente aquellas asociadas con la hospitalidad ritual, como cucharas (el énfasis es del autor), tazones bien elaborados y decorados. La cerámica cajamarquina fue también incluida en ofrendas rituales, como la de Ayapata (Ravines 1968, 1977). La extensa distribución y el contexto ritual en el que se encuentra la cerámica Cajamarca es un indicador de prestigio para esta cultura norteña" (Topic y Topic 2001: 186-187).

Los mismos autores interpretan en Marcahuamachuco «...los galpones nichados como ambientes dedicados a la veneración de los ancestros. Además de los huesos, se encontraron restos de comida y la cerámica aso- 
ciada tiene una mayor frecuencia en tazones, cuencos y cucharas (el énfasis es del autor) decoradas que aquella existente en otros contextos en Marcahuamachuco. La presencia de comida y vasijas sugiere que su colocación era precedida por actividades rituales dentro de un evento festivo" (Topic y Topic 2001: 189-191).

George Lau comenta que el intercambio más evidente entre Cajamarca y Ancash lo encuentra durante el Horizonte Medio, entre los años 600900 d.C. en tazones y cucharas de caolín. Las cucharas son pequeñas. Unos ejemplos de un entierro de Wilkawain y Carhuaz indican que estos objetos fueron reutilizados, con los bordes suavizados, no obstante el hecho que ya estaban rotos (Bennett 1944: $132 \mathrm{~d}, \mathrm{e}$ ).

Finalmente, y regresando al valle de Ayacucho, Juan Leoni (2004) nos ofrece un ejemplo del sitio de Nawinpukio, ubicado a pocos kilómetros al sur de Conchopata. Este sitio tiene una ocupación Huarpa tardío y del Horizonte Medio. Leoni encontró una ofrenda de cuatro cucharas huarpa. Esto confirma la importancia y el rol ceremonial de estos utensilios y discute si las cucharitas y cucharas fueron concebidas como cubiertos o más bien como vehículos para integrar a los difuntos con la comunidad y las huacas a través de la reciprocidad de elementos de consumo para el pueblo y los dioses.

Para resumir, tenemos datos de la sierra norte y de Ayacucho en la sierra central, que nos ofrecen unas nuevas perspectivas:

Las cucharitas y algunas cucharas de cerámica jugaron un rol importante en las actividades rituales en el norte durante el Periodo Intermedio Temprano y en Ayacucho. Todas las cucharas de Nawinpukio son de la época Huarpa.

Los cucharones no se encuentran en contextos de ofrendas o tumbas, sugiriendo que tenían una función más apropiada en la preparación de alimentos, mezcla y para medir quizás porciones iguales de comida en tazones de tamaños estándar. Los desgastes indican que fueron utilizados para la preparación y servicio de comidas. Las cucharas tenían huellas de desgaste en la parte que tocaba los lados y el fondo de una olla.

En general, las cucharas y cucharones se encuentran en áreas domésticas y áreas de preparación y consumo de alimentos en casas y patios donde ocurrieron festines. Estos son patrones generales de la distribución de cucharas y cucharones, los detalles serán materia de una futura publicación. 
Finalmente, una sugerencia muy especulativa. He observado que las proporciones de las cucharas combinan rasgos paralelos a las figurinas hechas de cerámica y otros materiales, como por ejemplo las turquesas de Pikillacta. Las cucharas tienen la parte distal larga y amplia en comparación al mango que muchas veces tiene un terminal modelado. Las figurinas tienen un cuerpo corto, como el mango de la cuchara, y la cara es siempre proporcionalmente más grande que el cuerpo. Los pies están en la posición terminal con un mínimo de modelación.

¿Pudiera ser que las cucharas que dan vida, porque llevan comida y bebida al cuerpo humano, eran concebidas como un ser humano?

Las cuatro cucharitas encontradas recientemente en Huari, en una ofrenda muy grande que incluía 365 vasijas en miniatura (González Carré y Soto 2004), tienen tres puntos en el diseńo interno de la cuchara misma, o la parte distal. Parecen ser dos ojos y una boca mirando y riéndose de nuestras interpretaciones a través de los siglos.

Agradecimientos: Quiero agradecer a Abelardo Sandoval por su apoyo, sus comentarios y ayuda con mi español humilde, por su amplio conocimiento de la arqueología peruana y como artista gráfico en las figuras de este artículo. Bárbara Wolff, estudiante doctoral de la Universidad Católica de América, ofreció su casa en Ayacucho durante su estadía para el análisis de materiales con apoyo de la Comisión Fulbright. Como parte de sus estudios doctorales, Bárbara está investigando en Conchopata, la organización de la producción, y quiénes fueron los productores de la cerámica de uso especial, como las urnas grandes, las jarras pintadas y las vasijas en miniatura. Su estudio sobre huellas de desgaste y las observaciones hechas por Oscar Huamán López relacionadas al proceso de producción, ayudaron en el reconocimiento de los posibles usos de las cucharas. Wolff también ha contribuido inmensamente a la creación de una base de datos en ACCESS de los hallazgos especiales, sin la cual este estudio no habría sido posible. También agradezco a los arqueólogos Lorenzo Huisa, quien empezó el análisis, y Oscar Huamán López, quien hizo gran parte del trabajo analítico. Sus observaciones, dibujos de posibles usos de cucharas e informes han contribuido significativamente a este estudio. Mis agradecimientos a Susana Arce por sus comentarios y por haber presentado la ponencia en Lima durante el simposio. Una persona que es realmente clave en nuestro proyecto es Jimmy Jáuregui, quien maneja, introduce y corrige los datos en la computadora, y ayuda en el manejo general de los asuntos administrativos del proyecto. 
Por último quiero reconocer a todos los miembros del Proyecto Arqueológico de Conchopata y al codirector William Isbell, porque realmente este trabajo y todo lo que estamos haciendo no hubiera sido posible sin la contribución de cada uno de ellos.

Queremos agradecer al apoyo financiero en forma de becas que el Proyecto Arqueológico Conchopata ha recibido de Dumbarton Oaks, National Geographic Society, National Science Foundation, Brennan Foundation y del Fondo de Investigaciones de la Facultad de la Universidad Católica de América en Washington, D. C.

Notas:

1 Visllacuni = «sacar algo con cuchara» (Holguín 1989 [1608]: 354)

2 Los estilos han sido definidos utilizando la cronología desarrollada localmente. Estamos en el proceso de refinar los estilos que se encuentran en las excavaciones, los que presentaremos en futuras publicaciones.

\section{BibliografíA}

Anders, M. 1986. Dual organization and calendars inferred from the planned site of Azangaro: Wari administrative strategies. Tesis doctoral. Department of Anthropology, Cornell University.

Benavides, M. 1965. Estudio de la cerámica decorada de Qonchopata, Ayacucho. Tesis de bachillerato. Universidad Nacional de San Cristóbal de Huamanga, Ayacucho.

Bennett, W. C. 1944. "The North Highlands of Peru: excavations in the Callejón de Huayllas and at Chavin de Huantar". Anthropological Papers of the American Museum of Natural History 39 (1): 1-114.

Brewster-Wray, C. C. 1983. "Spatial patterning and the function of a Huari architectural compound". En Investigations of the Andean Past, editado por D. H. Sandweiss, pp: 122-135. Cornell University. Latin American Studies Program, Ithaca.

Brewster-Wray, C. C. 1990. Moraduchayoq: an administrative compound at the site of Huari, Peru. Tesis doctoral. State University of Binghamton, New York.

Cook, A. G. y N. L. Benco. 2001. "Vasijas para la fiesta y la fama: producción artesanal en un centro urbano huari”. Boletín de Arqueología PUCP 4: 489-504. 
Cook, A. G. y M. Glowacki. 2003. "Pots, politics, and power: Huari ceramic assemblages and imperial administration". En The archaeology and politics of food and feasting in early states and empires, editado por T. L. Bray, pp: 173-202. Springer, New York.

Espoueys, O. 1972-1973. "Tipificación de cucharas de madera de Arica”. Actas del VI Congreso de Arqueología Chilena, pp: 63-109. Santiago.

Gero, J. M. 2001. "Field knots and ceramic beaus: Interpreting gender in the peruvian Early Intermediate Period". En Gender in pre-hispanic America, editado por C. F. Klein y J. Quilter, pp: 15-55. Dumbarton Oaks, Washington DC.

González Carré E. y J. Soto Maguino. 2004. Una ofrenda wari. Cuaderno de Investigación, Serie Arqueología No 2. Museo Nacional de Arqueología, Antropología e Historia del Perú, Lima.

Gonzales Holguín, D. 1989 [1608]. Vocabulario de la Lengua General de todo El Perú llamada Lengua Qquichua o del Inca. Universidad Nacional Mayor de San Marcos, Lima.

Gosden, C. e Y. Marshal. 1999. "The cultural biography of objects". World Archaeology 31(2): 169-178.

Huamán López, O. 2005. Informe preliminar de análisis de cucharas del sitio arqueológico de Conchopata. Proyecto Arqueológico Conchopata, Ayacucho.

Isbell, W.H. 2001. "Repensando el Horizonte Medio: el caso de Conchopata, Ayacucho, Perú". Boletín de Arqueología PUCP 4: 9-68.

Isbell, W. H. 2004. "Mortuary preferences: A Wari culture case study from the Middle Horizon Peru". Latin American Antiquity, 15 (1): 3-32.

Isbell, W. H. y A. G. Cook. 2002. "A new perspective on Conchopata and the Andean Middle Horizon". En Andean Archaeology II. Art, Landscape, and Society, editado por H. Silverman y W. H. Isbell, pp: 249-305. Kluwer Academic / Plenum Publishers, New York.

Isbell, W. H.; A. G. Cook, A. Carvajal, J. Ochatoma, C. Milliken y M. Cabrera Romero. 2002. Informe final al Instituto Nacional de Cultura del Perú del Proyecto Arqueológico Conchopata, Año 2001-2000, Vol. 1. INC, Ayacucho.

Leoni, J. B. 2004. Ritual, place, and memory in the construction of community identity: a diachronic view from Nawinpukio (Ayacucho, Peru). Tesis 
doctoral. Department of Anthropology, State University of New York. Binghamton.

Lumbreras, L. G. 1974. Las Fundaciones de Huamanga. Hacia una prehistoria de Ayacucho. Editorial Nueva Educación, Lima.

Ochatoma, J. y M. Cabrera. 2000. Excavaciones en un poblado alfarero de la época Huari. Manuscrito enviado al Instituto Nacional de Cultura, Lima.

Olivas Weston, R. 2001. La cocina de los incas: Costumbres gastronómicas y técnicas culinarias. Universidad San Martín de Porres, Lima.

Pérez Calderón, I. 1998. "Excavación y definición de un taller de alfareros Huari en Conchopata". Conchopata. Revista de Arqueología 1: 92137.

Pérez Calderón, I. y J. Ochatoma Paravicino. 1998. "Viviendas, talleres y hornos de producción alfarera Huari en Conchopata". Conchopata, Revista de Arqueología 1: 73-92.

Pozzi-Escott, D. 1991. "Conchopata: a community of potters". En Huari Administrative Structure: Prehistoric Monumental Architecture and State Government, editado por W. H. Isbell y G. F. McEwan, pp: 8192. Dumbarton Oaks, Washington D.C.

Topic, J.y T. Topic. 2001. "Hacía la comprensión del fenómeno huari: una perspectiva norteña”. Boletín de Arqueología PUCP 4: 181-217.

Topic, T y J. Topic. 1984. Huamachuco Archaeological Project: Preliminary Report on the First Season, June-August 1983. Occasional Papers in Anthropology No 1 . Trent University, Peterborough.

Tung, T. A. 2003. A bioarchaeological perspective on Wari Imperialism in the Andes of Peru: A view from heartland and hinterland skeletal populations. Tesis doctoral. Department of Anthropology, University of North Carolina Chapel Hill. Chapel Hill, NC.

Tung, T. A. y A. G. Cook. 2006. "Intermediate elite agency in the Wari Empire: the bioarchaeological and mortuary evidence". En Between King and Commoner: Intermediate Elites in Pre-Columbian States and Empires, editado por C. M. Elson y R. Alan Covey, pp 68-93. University of Arizona Press. 